\title{
Efeito de Sêmen Resfriado e Diluído em Beltsville Thawing Solution, Zorlesco-modificado e BTZOR no Desempenho Reprodutivo de Fêmeas Suínas Inseminadas
}

\author{
Ana Maria Martins Alves Vasconcelos ${ }^{2}$, Gentil Vanini de Moraes $^{3}$, Luís Paulo Rigolon ${ }^{3}$, \\ Ivan Moreira ${ }^{3}$, Elias Nunes Martins ${ }^{4}$
}

\begin{abstract}
RESUMO - Utilizaram-se 83 fêmeas, inseminadas com ejaculados de três cachaços, diluídos em Beltsville Thawing Solution (BTS), Zorlesco-modificado (ZOR) e BTZOR (meio desenvolvido na Universidade Estadual de Maringá), sendo 28 fêmeas inseminadas com o diluente BTS, 28 com ZOR e 27 com BTZOR, com o objetivo de estudar os índices de retorno ao cio e de parição e os números médios de leitões nascidos e nascidos vivos das fêmeas inseminadas. As fêmeas inseminadas com ZOR apresentaram melhor índice de parição (92,9\%), quando comparadas às inseminadas com BTZOR (70,4\%) e BTS $(67,9 \%)$. O índice de retorno ao cio foi menor nas fêmeas inseminadas com ZOR (7,14\%) em comparação às inseminadas com BTZOR (29,6\%) e BTS (32,14\%), não tendo diferença entre o BTS e o BTZOR. Concluiu-se que os melhores resultados quanto ao desempenho reprodutivo das fêmeas ocorreram nas inseminações realizadas com sêmen diluído em Zorlesco-modificado.
\end{abstract}

Palavras-chave: desempenho reprodutivo, diluentes, fêmeas suínas, inseminação artificial

\section{Reproductive Performance of Swine Females Inseminated with Cooled and Diluted Semen In Beltsville Thawing Solution, Modified-Zorlesco and BTZOR}

\begin{abstract}
Eighty-three swine females were inseminated with semen from three boars, diluted in Beltsville Thawing Solution (BTS), Modified-Zorlesco (ZOR) and BTZOR (a medium developed at Universidade Estadual de Maringá). Twenty eight females were inseminated with BTS, 28 with ZOR and 27 with BTZOR, with the objective to evaluate the indexes of heat return and farrowing rate, and the averages numbers of born piglets and born alive piglets from the inseminated females. The females inseminated with ZOR showed the best farrowing rate index (92.9\%) when compared to those inseminated with BTZOR $(70,4 \%)$ and with BTS (67.9\%). The lowest heat return rate was from inseminated females with ZOR (7.14\%) when compared to BTZOR (29.6\%) and BTS (32.14\%), and there was no difference between BTS and BTZOR. The best reproductive performance results were observed for artificial inseminations with semen diluted in Modified-Zorlesco diluents.
\end{abstract}

Key Words: diluents, reproductive performance, swine females artificial insemination

\section{Introdução}

A busca por um diluente que proporcione bons resultados na inseminação artificial (IA) de suínos tem sido uma constante dos pesquisadores desta área. BARITEAU et al. (1977) afirmaram que o uso do sêmen diluído na IA simplifica as operações desta técnica e não diminui os resultados de fecundidade média, o que também está de acordo com ZAVOS e LIPTRAP (1987), que observaram fecundidade e leitegadas iguais ou melhores que as obtidas por monta natural ou com sêmen congelado (SCHEID et al. 1986).

SENEGACNIK (1976), testando a resposta de sêmen resfriado em 2000 porcas, verificou que o índice de concepção para uma ou duas inseminações, com sêmen conservado de 1 a 24 horas, variou de 66,12 a 70,50\% para vários diluentes. JOHNSON et al. (1979), ao realizarem inseminação com sêmen resfriado e diluído em Kiev, no primeiro dia de colheita do sêmen, obtiveram $79 \%$ de parição, com a média de 10,6 leitões por leitegada.

GOTTARDI et al. (1980) inseminaram fêmeas com sêmen diluído em Zorlesco, cujo período de conservação variou de 1 a 4 dias, e obtiveram índices de parições que variaram de 58,5 a 78,7\% e leitegadas, que variaram de 8,5 a 9,8 leitões, enquanto GOMES et al. (1982), utilizando sêmen diluído em Kiev (semelhante ao BTS), de um dia de conservação, obtiveram $83,3 \%$ de parição e leitegadas médias de 9,5 leitões,

\footnotetext{
${ }_{1}^{1}$ Parte da dissertação de Mestrado da primeira autora apresentada à UEM.

${ }^{2}$ Enga -Agrônoma, Mestre em Zootecnia. E.mail: amavasco@terra.com.br

${ }^{3}$ Professor do DZO da Univesidade Estadual de Maringá/UEM. E.mail: gvmoraes@uem.br; rig@wnet.com.br; imoreira@uem.br

${ }^{4}$ Professor do DZO/UEM e pesquisador do CNPq. E.mail: enmartins@uem.br
} 
mostrando que o Zorlesco, mesmo com maior tempo de conservação apresentou as mesmas médias de leitões. Por outro lado, JOHNSON et al. (1982a) verificaram redução de fecundidade, quando as fêmeas foram inseminadas com sêmen conservado por mais de dois dias. Porém, GOMES et al. (1982), trabalhando com 13 matrizes obtiveram $84,5 \%$ de índice de concepção e média de 9,5 leitões/leitegada, ao utilizarem sêmen resfriado e diluído em meio Kiev.

PAQUIGNON et al. (1987) verificaram que fêmeas inseminadas com sêmen diluído em BTS, cujo período de conservação foi de quatro dias, apresentaram índices de prenhez que variaram de $73,1 \%$ (1음 dia) a $68,0 \%$ (4음 dia) com 11,0 a 10,8 leitões/ leitegada, respectivamente.

VIEIRA (1988) reconheceu que houve progresso na difusão da técnica de IA com duas doses inseminantes por fêmea, melhorando a fertilidade e a prolificidade, registrando-se valores médios de apenas um retorno ao cio para cada 20 porcas inseminadas com sêmen diluído em diluente de alta qualidade. REVELL e GLOSSOP (1989) notaram boa resposta reprodutiva das fêmeas, quando o sêmen e o diluente foram de alta qualidade, uma vez que as experiências mostrarem que apenas $20 \%$ dos ejaculados coletados de estudos típicos de IA poderiam ser conservados por longo tempo. Constataram, também, diferenças entre diluentes quanto à fecundidade, destacando a necessidade de se descobrirem diluentes capazes de conservar sêmen por cinco a seis dias, sem que haja redução na fecundidade das fêmeas, ao serem inseminadas.

TONIOLLO e MESQUITA (1990) mostraram que sêmen de suíno, ao ser diluído em água de côco estabilizada, apresentou baixo índice de retorno ao cio, devido ao fato de os espermatozóides terem encontrado bom meio de sobrevivência, o que lhes conferiu maior eficácia no trato genital feminino, fecundando maior número de óvulos.

PEDERSEN et al. (1995) inseminaram porcas com sêmen conservado em BTS ou Kiev/EDTA e verificaram que o tamanho da leitegada e o índice de parição não foram afetados por doses inseminantes contendo dois bilhões de espermatozóides comparadas às doses inseminantes contendo quatro bilhões de espermatozóides. Porém, de acordo com PIG INTERNATIONAL (1994), não se sabe a concentração espermática exata necessária à dose de sêmen para a obtenção de bons resultados quanto à fecundação e ao número de leitões nascidos por leitegada.

BARITEAU et al. (1984) notaram que fêmeas inseminadas com doses de três bilhões espermatozóides, em que o sêmen foi diluído em BL1 e Guelph, ambos de composição semelhante à do BTS, conservados por dois dias, apresentaram índices de parição de 72,0 e 73,2\%, respectivamente; porcas inseminadas com uma dose de três bilhões de espermatozóides, diluídos em Guelph, apresentaram $73,9 \%$ de parição e 10,5 leitões/leitegada com zero dia de conservação e $72,4 \%$ de parição e 10,2 leitões/ leitegada com um dia de conservação, mostrando que o tempo de conservação e o número de espermatozóides por dose inseminante influenciam essas respostas reprodutivas.

TONIOLLO e MESQUITA (1990) usaram BTS e água de côco estabilizada como diluentes de sêmen suíno, com a finalidade de avaliar a fertilidade das fêmeas, tendo constatado índices de parição de 74,37 e $86,45 \%$, respectivamente, dentro de um mesmo período de tempo. BORTOLOZZO et al. (1993) utilizaram BTS, com doses inseminantes de três bilhões de espermatozóides e uma única inseminação, com sêmen conservado por 1, 2 e 3 dias e constataram, após abate das fêmeas, que os índices de fertilização foram de 77,4; 77,3 e 72,4\%, respectivamente.

WABERSKI et al. (1994), estudando o potencial de fecundação do sêmen conservado por cinco dias, diluído no meio BTS, observaram que os índices de fecundidade referentes a cada dia foram 87,2;68,9, 75,$6 ; 61,4 ; \mathrm{e} 31,9 \%$, respectivamente. ALEXOPOULOS et al. (1996) utilizaram sêmen de cachaços conservado por 48 e 72 horas, diluído em BTS, em que realizaram duas inseminações por fêmea, com doses inseminantes de um, três e cinco bilhões de espermatozóides. Os melhores índices de retorno ao cio e de parições foram observados nas doses com um e três bilhões de espermatozóides em relação às doses com cinco bilhões.

O objetivo desta pesquisa foi estudar os efeitos dos diluentes Beltsville Thawing Solution (BTS), Zorlescomodificado (ZOR) e BTZOR (meio desenvolvido na Universidade Estadual de Maringá) sobre os índices de retorno ao cio, parição e tamanho de leitegada.

\section{Material e Métodos}

O experimento foi conduzido na Fazenda Experimental da Universidade Estadual de Maringá (UEM), em Maringá-PR, de maio de 1996 a maio de 1997. Foram utilizadas, em média, sete fêmeas por mês, entre porcas e marrãs. Os animais foram mantidos em instalações convencionais para a criação de suínos, do tipo semi-confinada. As fêmeas receberam duas 
404 Rev. bras. zootec.

refeições de 1,8 a 2,0 kg de ração de gestação por dia, contendo $13,55 \%$ de PB e 3,02 Mcal de energia metabolizável por quilo de ração, além de núcleo mineral e vitamínico suiplus (Nutris).

As fêmeas que apresentaram reflexo de monta ao inseminador, que, em média, ocorreu quatro dias após o desmame, foram inseminadas e, em seguida, conduzidas a uma baia contendo oito gaiolas individuais. As marrãs, ao serem incluídas nos grupos das porcas, tiveram cio induzido mediante a aproximação de cachaços, rufiões e realização de caminhadas. A inseminação foi feita sempre pelo mesmo inseminador, que usou um catéter por fêmea por inseminação. Foram realizadas duas inseminações por cio, sendo a primeira, em média, 36 horas após o início do cio, quando as fêmeas apresentaram reflexo de monta ao inseminador e a segunda inseminação de 8 a 12 horas após a primeira. A quantidade total média de espermatozóides, por dose inseminante, foi de cinco bilhões, dentro dos limites apontados como excelentes por SCHEID et al. (1993). As fêmeas inseminadas foram observadas, individualmente, por 25 dias, para a detecção de retorno ao cio e aquelas prenhes foram colocadas numa área com piquetes de terra e baias. Uma semana antes da data prevista para as parições, as fêmeas foram transferidas para a maternidade e submetidas a um banho completo. As everminações foram feitas 15 dias antes do parto, uma semana antes do parto e oito dias após o desmame, tendo os vermífugos sido administrados junto à ração.

Nas fêmeas inseminadas, avaliaram-se os índices de retorno ao cio e de parição e os números médios de leitões nascidos e nascidos vivos por leitegada por fêmea. O experimento foi conduzido utilizando-se três diluentes e três cachaços, durante 12 meses. Foram utilizadas 83 fêmeas entre porcas e marrãs, sendo inseminadas 28 fêmeas com sêmen diluído em BTS, 28 em ZOR e 27 em BTZOR, tendo cada fêmea representado uma unidade experimental. A composição de cada diluidor encontra-se na Tabela 1 . O efeito do

Tabela 1 - Composição dos diluentes (BTS, Zorlesco - modificado e BTZOR)

Table 1 - Diluents composition (BTS, modified Zorlesco and BTZOR)

\begin{tabular}{|c|c|c|c|}
\hline $\begin{array}{l}\text { Produtos } \\
\text { Products }\end{array}$ & $\begin{array}{l}\operatorname{BTS}^{1}(\mathrm{~g}) \\
B T S^{1}(g)\end{array}$ & $\begin{array}{c}\text { Zorlesco modificado }^{2}(\mathrm{~g}) \\
\text { Modified Zorlesco } \\
(\mathrm{g})\end{array}$ & $\begin{array}{r}\mathrm{BTZOR}^{3}(\mathrm{~g}) \\
\operatorname{BTZOR}^{3}(\mathrm{~g}) \\
\end{array}$ \\
\hline Glicose & 37,00 & 11,50 & 24,00 \\
\hline Glucose & & & \\
\hline Citrato de sódio & 6,00 & 11,65 & 4,80 \\
\hline $\begin{array}{l}\text { Sodium citrate } \\
\text { Bicarbonato de sódio } \\
\text { Sodium bicarbonate }\end{array}$ & 1,25 & 1,75 & 1,50 \\
\hline $\begin{array}{l}\text { EDTA }^{4} \\
\text { EDTA }^{4}\end{array}$ & 1,25 & 2,35 & 1,80 \\
\hline $\begin{array}{l}\text { TRIS }^{5} \\
\text { TRIS }\end{array}$ & - & 6,50 & 3,50 \\
\hline $\begin{array}{l}\text { Cloreto de potássio } \\
\text { Potassium chloride }\end{array}$ & 0,75 & - & 0,40 \\
\hline $\begin{array}{l}\text { Penicilina G sódica } \\
\text { Penicilin G sodium }\end{array}$ & 1,00 & 1,00 & 1,00 \\
\hline $\begin{array}{l}\text { Diidrostreptoomicina } \\
\text { Dihydrostreptomycin }\end{array}$ & 1,00 & 1,00 & 1,00 \\
\hline $\begin{array}{l}\text { Ácido cítrico } \\
\text { Citric acid }\end{array}$ & - & 4,10 & 2,00 \\
\hline $\begin{array}{l}\text { Soro Fetal de Bovino (BSA -V) } \\
\text { Bovine Serum Albumin }(B S A-V)\end{array}$ & - & 5,00 & 3,00 \\
\hline $\begin{array}{l}\text { Cisteína } \\
\text { Cysteine }\end{array}$ & - & 0,07 & 0,04 \\
\hline $\begin{array}{l}\text { Água deionizada (1) } \\
\text { Deionized water }(l)\end{array}$ & 1,00 & 1,00 & 1,00 \\
\hline
\end{tabular}

1 BTS: Beltesville Thawing Solution (WOELDERS, 1992); ${ }^{2}$ Zorlesco modificado (MORAES e MOREIRA, 1995) (Modified-Zorlesco [MORAES e MOREIRA, 1995]); ${ }^{3}$ BTZOR: Meio desenvolvido no laboratório da UEM (MORAES e MOREIRA, 1995) (BTZOR: developed médium at UEM laboratory); ${ }^{4}$ EDTA: Etilenodiamino tetracetato (EDTA: ethylenediamine tetracetate); ${ }^{5}$ TRIS: Hidroxi-metil -aminometano (TRIS: Hydroxymethylaminomethane). 
macho está confundido com o efeito do mês propositalmente, já que o objetivo do principal era checar o efeito do diluente sobre o sêmen. Além disso, o número de fêmeas utilizado no experimento não permitia grande número de machos, inserido no modelo para tirar seu efeito. O número de leitões/leitegada foi submetido à análise de variância, utilizando-se o seguinte modelo: $\mathrm{Y}_{\mathrm{ijkl}}=\mu+\mathrm{D}_{\mathrm{i}}+\mathrm{B}_{\mathrm{j}}+\mathrm{C}_{\mathrm{k}}+\mathrm{b}\left(\mathrm{OP}_{\mathrm{ijkl}}-\overline{\mathrm{OP}}\right)+\mathrm{b}^{2}\left(\mathrm{OP}_{\mathrm{ijk}}-\overline{\mathrm{OP}}\right)^{2}+\mathrm{e}_{\mathrm{ijkl}}$ em que $Y_{i j k l}$ é observação referente à porca "l", inseminada com sêmen do cachaço "k", usando o diluente "i" no mês "j"; $\mu$, constante geral; $\mathrm{D}_{\mathrm{i}}$, efeito do diluente $\mathrm{i}, \mathrm{i}=1 ; 2 ; 3 ; \mathrm{B}_{\mathrm{j}}$, efeito do bloco $\mathrm{j}, \mathrm{j}=1 ; \ldots ; 12 ; \mathrm{C}_{\mathrm{k}}$, efeito do cachaço $\mathrm{k}, \mathrm{k}=1,2,3$; $\mathrm{OP}_{\mathrm{ijkl}}$, ordem de parto da porca "l" inseminada pelo cachaço "k", usando o diluente "ii” no mês “j”; $\overline{\mathrm{OP}}$, média observada das ordens de parto; $\mathrm{e}_{\mathrm{ijkl}}$, erro aleatório associado à cada observação $\mathrm{Y}_{\mathrm{ijkl}}$.

As diferenças entre os índices de prenhez e o retorno ao cio, para identificação de diferenças entre as médias foram analisados pelo teste exato de Fisher, $5 \%$, pelo programa de análise estatística SAS (1985).

\section{Resultados e Discussão}

Os resultados de porcentagens de retorno ao cio, índice de parição, número médio de leitões nascidos e número médio de leitões nascidos vivos por leitegada são apresentados na Tabela 2 .
O diluente ZOR que conservou o sêmen em condições de ser aproveitado na IA, em média, por 4,62 dias (VASCONCELOS et al., 2001), apresentou o melhor índice de parição $(92,9 \%)$, mostrando ser um meio viável à IA de suínos. Já o BTZOR, que, em média, conservou o sêmen por 4,94 dias, apresentou índice de parição de 70,4\% e o BTS, que, em média, conservou o sêmen por 3,27 dias, de 67,9\%. Talvez, o melhor resultado do ZOR esteja relacionado com sua composição, como por exemplo a albumina sérica de bovinos (fração V), que deve ter proporcionado melhores condições aos espermatozóides, em função de suas propriedades anti-aglutinantes e anti-tóxicas. Contudo, o BTZOR também tem albumina sérica de bovinos (fração V) na sua constituição, mas em valores menores $(40 \%)$ em relação ao ZOR, porém com resposta tendendo a ser superior a do BTS, que não tem albumina sérica em sua composição. O ZOR e o BTZOR têm outros ingredientes que o BTS não tem, como cisteína, ácido cítrico, TRIS (hidroximetil aminometano) e maior quantidade de citrato de sódio.

O número médio de leitões nascidos e o número médio de leitões nascidos vivos por leitegada foram maiores $(\mathrm{P}<0,05)$ de 11,89 e 10,37, quando se utilizou ZOR, àqueles observados no BTZOR de 9,2 e 7,33 e também aos observados no BTS, que foram de 8,97 e 7,55 leitões/leitegada, respectivamente. Não houve efeito de macho e de mês de IA, e sim efeito

Tabela 2 - Parâmetros médios de avaliação de fecundidade de fêmeas suínas inseminadas com sêmen suíno diluído em Beltsville Thawing Solution (BTS), Zorlesco-modificado e BTZOR (meio desenvolvido na Universidade Estadual de Maringá)

Table 2 - Average parameters of fecundity evaluation of swine females inseminated with swine semen diluted in Beltsville Thawing Solution (BTS), Modified Zorlesco and BTZOR (media developed at Universidade Estadual de Maringá)

\begin{tabular}{|c|c|c|c|}
\hline \multirow[b]{2}{*}{$\begin{array}{l}\text { Parâmetro } \\
\text { Parameter }\end{array}$} & \multicolumn{3}{|c|}{$\begin{array}{l}\text { Diluente } \\
\text { Diluent }\end{array}$} \\
\hline & $\begin{array}{l}\mathrm{BTS}(28) \pm \mathrm{EP} \\
B T S(28) \pm S E\end{array}$ & $\begin{array}{l}\text { Zorlesco }(28) \pm \mathrm{EP} \\
\text { Zorlesco }(28) \pm S E\end{array}$ & $\begin{array}{l}\text { BTZOR (27) } \pm \mathrm{EP} \\
B T Z O R(27) \pm S E\end{array}$ \\
\hline $\begin{array}{l}\text { Índice de retorno } \\
\text { ao cio }(\%)\end{array}$ & $32,1^{b}$ & $7,1^{\mathrm{a}}$ & $29,6^{b}$ \\
\hline $\begin{array}{l}\text { Return rate }(\%) \\
\text { Índice de parição(\%) }\end{array}$ & $67,9^{\mathrm{b}}$ & $92,9^{\mathrm{a}}$ & $70,4^{b}$ \\
\hline $\begin{array}{l}\text { Farrowing rate }(\%) \\
\text { Média de leitões/leitegada } \\
\text { Average of piglets born/litter }\end{array}$ & $8,97 \pm 1,48^{b}$ & $11,89 \pm 1,48^{\mathrm{a}}$ & $9,18 \pm 1,51^{b}$ \\
\hline $\begin{array}{l}\text { Média de leitões vivos/leitegada } \\
\text { Average of piglets born alive/litter }\end{array}$ & $7,55 \pm 1,48^{b}$ & $10,37 \pm 1,48^{a}$ & $7,33 \pm 1,51^{b}$ \\
\hline
\end{tabular}

Valores com sobrescritos diferentes, nas linhas, diferem $(P<0,05)$.

( ) número de observações.

$\mathrm{EP}=$ erro-padrão.

Values followed by different superscripted, within rows, are different $(P<.05)$.

() number of observations.

$S E=$ standard error 
quadrático $(\mathrm{P}<0,05)$ da ordem de parto em relação ao tamanho da leitegada, observando-se aumento no número de leitões até a oitava ordem de parto e decréscimo no número de leitões a partir deste momento. O índice de retorno ao cio foi menor $(\mathrm{P}<0,05)$ nas fêmeas inseminadas com ZOR em relação àquelas inseminadas com sêmen diluído em BTZOR e BTS (Tabela 1).

Os resultados de $76,7 \%$ de parição obtidos por GOTTARDI et al. (1980), ao estudarem o diluente Zorlesco na IA de suínos, em que o sêmen foi conservado por quatro dias, foram inferiores aos obtidos neste experimento. Os mesmos autores, testando sêmen diluído e conservado em Zorlesco por diversos dias ,obtiveram 69,8; 62,5 e 43,0\% de parições, para sêmen conservado por cinco, seis e oito dias, respectivamente. Perez Lys et al. (1984), citados por WEITZE (1990), ao conservarem sêmen diluído em MR-A e Zorlesco, entre sete e nove dias e em Kiev por três dias, concluíram que os índices de parições, nos respectivos dias de conservação, foram de $73,2 \%$ para o MR-A, 59,0\% para o Zorlesco e 80,5\% para o Kiev, notando-se que tais resultados foram inferiores aos encontrados neste experimento, provavelmente, devido ao maior período de conservação do sêmen e às características químicas dos diluentes, posto que, neste experimento, as fêmeas foram inseminadas com o ZorlescoModificado à base de penicilina $\mathrm{G}$ sódica, que permitia melhor e maior conservação do mesmo e, conseqüentemente, melhor índice de parição. JOHNSON et al. (1982b), ao diluírem sêmen em meio Kiev, verificaram que 65,9 e 52,7\% das 2849 fêmeas inseminadas ficaram prenhes com sêmen de um e três dias, respectivamente, observando-se redução significativa no índice de prenhez em relação aos dias decorridos, sendo estes resultados inferiores aos $67,9 \%$ obtidos neste experimento. $\mathrm{O}$ período de experimento da pesquisa dos autores foi de 8 semanas, sendo administradas doses inseminantes de $100 \mathrm{~mL}$ com sêmen de um dia de conservação e $25 \mathrm{~mL}$ com três dias.

Em relação ao ZOR e ao BTS, os resultados quanto aos índices de parição, aqui obtidos (Tabela 1) diferem daqueles obtidos por AALBERS et al. (1983) e JOHNSON et al. (1988), que mostraram resultados com sêmen conservado por três dias em BTS (75\%) superiores ao Zorlesco (65\%), em várias inseminações. Para estes autores, este decréscimo é resultante de interação significativa entre diluente e tempo de conservação, o que acredita-se estar também relacionado a este experimento.

AALBERS et al. (1983) conduziram dois experi- mentos em que avaliaram ejaculados de cachaço, conservados a $18{ }^{\circ} \mathrm{C}$ por três dias, os quais foram diluídos em BTS, Kiev e Zorlesco, verificaram que os índices de parições foram, respectivamente, de 74,6; 71,4 e $65,2 \%$, resultados que também são inferiores aos obtidos com o Zorlesco neste experimento. Essa diferença talvez resida na metodologia adotada nesta pesquisa, em que os estudos foram realizados durante um ano, em um único local, com menor número de porcas (83), mas com maior número de espermatozóides por dose inseminante, além de ter sido utilizado sêmen desde o dia um de conservação a $15{ }^{\circ} \mathrm{C}$. Já os autores acima utilizaram sêmen com somente três dias de conservação, tendo inseminado 900 porcas em seis diferentes locais.

AALBERS et al. (1984) trabalharam durante um ano e verificaram que porcas inseminadas com sêmen diluído em BTS, cujo período de conservação foi de um e de dois dias, apresentaram diferença $(\mathrm{P}<0,05)$ no tamanho da leitegada $(11,6$ vs 11,4), apesar de não ter ocorrido o mesmo para o índice de parição. Entretanto, esses autores notaram que porcas inseminadas com sêmen conservado por 1,5 dias tiveram tamanho da leitegada de 11,3 leitões e índice de parição de $82,6 \%$ contra 11,1 leitões/leitegada e $78,5 \%$ de parição com inseminações, cujo período de conservação do sêmen foi de 2,5 dias. Pode-se atribuir a essa resposta o fato de cada fêmea foi inseminada, em média, 6,5 vezes ao dia.

SILVEIRA (1983), trabalhando com o diluente Kiev, meio semelhante ao BTS e um sistema de uma ou duas inseminações, não constatou diferença na taxa de parição, porém, o número de leitões nascidos vivos com uma inseminação artificial foi, em média, de 10 leitões contra 10,63 com duas inseminações, tendo o autor obtido a média, entre os dois sistemas de inseminações, de $82,4 \%$ de parições. Estes dados foram superiores aos encontrados com BTS neste experimento (Tabela 2). Possivelmente, o menor índice de parição $(67,9 \%)$ e o menor número de leitões nascidos vivos por leitegada $(8,97)$, mostrado neste estudo, se devam ao menor número de fêmeas inseminadas durante o experimento (83). Outro fator que pode ser utilizado para justificar essas diferenças é que nesta pesquisa foi utilizado sêmen por mais que três dias de conservação, enquanto os referidos pesquisadores utilizaram sêmen conservados até o terceiro dia. Nesse sentido, BARITEAU et al.(1984) encontraram resultados semelhantes, tendo mencionado que o tempo de conservação e o número de espermatozóides por dose inseminante influenciam 
as respostas reprodutivas. Dados desses autores foram também semelhantes aos obtidos por TONIOLO e MESQUITA (1990) e BORTOLOZZO et al. (1993), que usaram o meio diluente BTS, mas com doses inseminates contendo 3 bilhões de espermatozóides e uma única inseminação.

PAQUIGNON et al. (1987) compararam os diluentes BTS e Guelph, quanto à fertilidade e à prolificidade das fêmeas, tendo notado melhor resultado com sêmen diluído em BTS. No sêmen diluído em Guelph e conservado por quatro dias, tendo o dia da colheita como "dia zero", os autores verificaram índices de prenhez de 73,$1 ; 74,9 ; 72,9$ e $55,8 \%$ e prolificidade de 10,9; 10,4;11,1 e 9,4 leitões/leitegada, nos respectivos dias. Quanto ao BTS, os índices de prenhez nos dias $0,1,2$ e 3 foram de 74,1; 77, $1 ; 76,9$ e $68,0 \%$, sendo a prolificidade de 11,$0 ; 10,9 ; 11,0 \mathrm{e}$ 10,8 leitões por leitegada, respectivamente. Estes resultados foram superiores aos obtidos nesta pesquisa com sêmen diluído em BTS (Tabela 1), considerando a faixa de conservação de três dias, mas os autores trabalharam com 925 porcas e duas doses de sêmen com $3 \times 10^{9}$ espermatozóides. Já nesta pesquisa, foram utilizadas duas inseminações e cada dose inseminante com $5 \times 10^{9}$ espermatozóides, além de terem sido inseminadas apenas 83 porcas. Por outro lado, BLICHFELDT et al. (1988) mostraram resultados de fêmeas inseminadas com BTS que tiveram taxas de concepção de 72,0; 75, 1 e 63,5\%, sendo a conservação do sêmen por um, dois e três dias, respectivamente, resultados estes semelhantes aos do BTS neste estudo. JOHNSON et al. (1988), ao trabalharem com porcas inseminadas com sêmen conservado no meio BTS, observaram 79,3\% de parição para sêmen conservado de um a quatro dias e total médio de 11,4 leitões/leitegada, em que as doses inseminantes continham 6 × $10^{9}$ espermatozóides. Além dissso, o trabalho foi desenvolvido, na Holanda, com 2896 porcas.

Resultados semelhantes aos deste estudo, em relação ao BTS, foram observados por WABERSKI et al. (1994) e ALEXOPOULOS et al. (1996), com sêmen conservado de dois a cinco dias e doses inseminantes de um a cinco bilhões de espermatozóides.

A respeito dos índices de parição, de retorno ao cio, do número de leitões nascidos e do número de leitões nascidos vivos por leitegada, o BTZOR apresentou comportamento com tendência a ser melhor que o BTS, porém inferior ao ZOR (Tabela 2), mas não se dispõe de outras informações para tecer discussões. De qualquer forma, foi um meio que apresentou resultados considerados razoáveis, indi- cando sua viabilidade de uso na IA de suínos. Ao considerar-se retorno ao cio (Tabela 2), registrou-se retorno de $32,1 \%$ para o BTS, enquanto TONIOLLO e MESQUITA (1990), em trabalho semelhante, verificaram $23,85 \%$ de retorno ao cio também com BTS, diferenças que talvez possam ser atribuídas à duração do experimento, que aqui foi de doze meses, sendo contempladas as variabilidades ambientais de todas as estações do ano. Já o BTZOR (29,6\%) apresentou-se melhor que o BTS $(32,1 \%)$, mas inferior ao ZOR $(7,1 \%)$, não se dispondo de informações concretas para maiores discussões. Contudo, podese especular sobre a composição dos diluidores, pois o BTZOR e o ZOR contêm em suas formulações (Tabela 1) albumina sérica de bovinos, fração $\mathrm{V}$, mais cisteína, o que pode ter conferido melhor qualidade aos esprmatozóides durante a conservação, o que poderia ter proporcionado melhores possibilidades para a fecundação.

\section{Conclusões}

As fêmeas inseminadas com sêmen diluído em Zorlesco-modificado apresentaram melhor índice de parição, menor índice de retorno ao cio, maior número médio de leitões nascidos e maior número médio de leitões nascidos vivos por leitegada em relação àquelas inseminadas com sêmen diluído BTS e BTZOR.

\section{Referências Bibliográficas}

AALBERS, J.G., RADEMAKER, J.H.M., GROOTEN, H.J.G. et al. 1983 Fecundity of boar semen stored in BTS, Kiev, Zorlesco and Modena extenders under field conditions. J. Anim. Sci., 57(suppl.1):314-315.

AALBERS, J. G., JONHSON, L.A., RADEMAKER, J.M.H. et al. Use of boar spermatozoa for AI: fertility and morphology of semen diluted in BTS and used for insemination within 24 hrs or 25 to $48 \mathrm{hrs}$ after collection. In: INTERNATIONAL CONGRESS ON ANIMAL REPRODUCTION AND ARTIFICIAL INSEMINATION, 10, 1984, Urbana, Champaign. Anais... Urbana, Champaign, 1984. v.2, n.180, p.360-362.

ALEXOPOULOS, C., BOSCOS, C., SARATSIS, C. et al. 1996. The effect of storage time and number of spermatozoa per insemination dose on semen characteristics and fertilizing capacity of boar semen diluted with Beltsville Thaw Solution (BTS) extender. Anim. Sci., 62(3):599-604.

BARITEAU, F., BUSSIERE, J., COUROT, M. et al. 1977. Insemination artificielle porcine ameliorations techniques, resultats recents. J. Rech. Porc. en France, 1:11-14.

BARITEAU, F., BUSSIERE, J., COUROT, M. et al. 1984. Insemination artificielle porcine. Mise en place par l'eleveur ou para l'inseminateur. J. Rech. Porcine en France, 16:173-179.

BLICHFELDT, T., ALMLID, T., STAUNE, S.E. Liquid preservation of boar semen in Kiev or BTS. A field comparison. In: INTERNATIONAL CONGRESS ON ANIMAL 
408 Rev. bras. zootec.

REPRODUCTION AND ARTIFICIAL INSEMINATION, 11, 1988, Ireland. Anais... Ireland, 1988. v.3, n.231, 3p.

BORTOLOZZO, F.P., LÜBBERT, Z.L.W., WABERSKY, D. et al. Armazenamento da dose espermática "in vitro" por 1, 2 e 3 dias e seus efeitos sobre a taxa de fertilização e número de espermatozóides acessórios após a inseminação artificial no suíno. In: CONGRESSO BRASILEIRO DE VETERINÁRIOS ESPECIALISTAS EM SUÍNOS, 6, Goiânia, 1993. Anais... Goiânia: CBVES, 1993. p.120.

GOMES, S.Z., TORRES, C.A., COSTA, P.M.A. 1982. Efeito da inseminação artificial com sêmen diluído (diluente Kiev) resfriado e sêmen congelado pelo método Hülsem-Berg/ Hannover sobre a eficiência reprodutiva das porcas. $R$. Soc. Bras. Zootec., 11(3):360-374.

GOTTARDI, L., BRUNEL, L., ZANELLI, L. New dilution media for artificial insemination in pig. In: INTERNATIONAL CONGRESS ON ANIMAL REPRODUCTION AND ARTIFICIAL INSEMINATION, 9, 1980, Madrid. Anais... Madrid, 1980. v.5, p.49.

JOHNSON, L.A., AALBERS, J.G., WILLIEMS, C.M.T. et al. 1979. Effectiveness of fresh and frozen boar semen under practical conditions. J. Anim. Sci., 49(suppl. 1):306.

JOHNSON, L.A., AALBERS, J.G., ARTS, J.A.M. 1982a. Use of boar spermatozoa for artificial insemination. II. Fertilizing capacity of fresh and frozen spermatozoa in gilts inseminated either at a fixed time or according to walsmeta readings. J. Anim. Sci., 54(1):126-131.

JOHNSON, L.A., AALBERS, J.G.,WILLEMS, C.M.T. et al. $1982 b$. Use of boar spermatozoa for artificial insemination. III. Fertilizing of boar spermatozoa stored in Beltsville Liquid and Kiev extenders for three days at $18^{\circ} \mathrm{C}$. J. Anim. Sci., 54(1):132-141.

JOHNSON, L.A., AALBERS, J.G., GROOTEN, H.J.G. 1988. Artificial insemination of swine: fecundity of boar semen stored in Beltsville TS (BTS), modified modena (MM), or MR-A and inseminated on one, three and four days after collection. Zuchthygiene, 23(2):49-55.

MORAES, G.V., MOREIRA, I. Diluentes na conservação de sêmen resfriado de suíno. In: REUNIÃO ANUAL DA SOCIEDADE BRASILEIRA DE ZOOTECNIA, 32, 1995, Brasília. Anais... Brasília: SBZ, 1995. p.450-451.

PAQUIGNON, M., BUSSIERE, J., BARITEAU, F. et al. Use of Guelph extender in the storage of boar spermatozoa. Limit of system. In: INTERNATIONAL CONGRESS ON ANIMAL REPRODUCTION AND ARTIFICIAL INSEMINATION, 10, 1984, Illinois. Anais... Illinois, 1984. 3p.

PAQUIGNON, M., BUSSIERE, J., BARITEAU, F. 1987. Resultats recents en matiere de technologie de la conservation de la semence de verrat. J. Rech. Porc. en France, 19:63-78.

PEDERSEN, N.P. THE INTERNATIONAL COMMITTEE FOR PIG BREEDING, HEALTH AND PRODUCTION et al. Handling semen. In: CONFERÊNCIA INTERNACIONAL SOBRE CIÊNCIA E TECNOLOGIA DE PRODUÇÃO E INDUSTRIALIZAÇÃO DE SUÍNOS, 1, 1995, Campinas. Anais...Campinas, 1995. p.65-69.
PIG INTERNATIONAL. 1994. Testing semen for quality. Pig Int.l, 24(5):15-16.

REVELL, S.G., GLOSSOP, C.E. 1989. A long-time ambient temperature diluent for boar semen. Anim. Prod., 48:579-584.

SAS. 1985. SAS User's Guide: Statistics (Version 5 Ed.). SAS int. Inc., Cary, NC.

SCHEID, I.R., WENTZ, I., MARIANO, M.S. 1986. Resultados comparativos da inseminação artificial em suínos com sêmen congelado e resfriado. Concórdia: EMBRAPA/CNPSA. 3 p (Comunicado técnico, 100).

SCHEID, I.R., WENTZ, I., FIALHO, F.B. et al. Fertilidade com sêmen suíno conservado nos diluentes Kiev, BTS, Reading. In: CONGRESSO BRASILEIRO DE VETERINÁRIOS ESPECIALISTAS EM SUÍNOS, 6, 1993, Goiânia. Anais... Goiânia: CBVES, 1993. p.117.

SENEGACNIK, J. 1976. The effect of some biochemical factors on the motility and fertility of fresh and deep-frozen semen in the boar. Dtsch. Tierarztl. Wochenschr., 83(12):537-539.

SILVEIRA, P.R.S. 1983. Efeito do diluente Kiev sobre a fertilidade de porcas inseminadas artificialmente. Santa Maria: UFSM. 9p (Seminário).

TONIOLLO, R., MESQUITA, D.S.M. 1990. Fertilidade de porcas inseminadas com sêmen diluído em água de côco estabilizada e com B.T.S. R. Bras. Reprod. Anim., 14(4):249-254.

VASCONCELOS, A.M..M.S., MORAES, G.V., RIGOLON, L.P. et al. 2001. Características espermáticas de sêmen resfriado de suíno e conservado em diferentes diluentes. Rev. bras. zootec., 30(2):385-392.

VIEIRA, H.P. 1988. Inseminação artificial porcina com sémen refrigerado, resultados práticos e interesse do método. R. Port. Ciên. Vet., 83:487.

WABERSKI, D., WEITZE, K.F., LIETMANN, C. et al. 1994. The initial fertilizing capacity of longterm-stored liquid boar semen following pre-and post ovulatory insemination. Theriogenology, 41(7):1367-1377.

WEITZE, K.F. 1990. The use of "Long-term Extender" in pig A.I. - a view of the international situation. Pig News and Inf., 11(1):23-26.

WOELDERS, H. 1992. Maintaining quality of boar sperm during storage and transportation. Pigs Misset, 8:22-23.

ZAVOS, E.D.S., LIPTRAP, D.O. 1987. Procedures for collection, evaluation, dilution and artificial insemination of boar spermatozoa. Agri-Pract., 8(3):19-23.

Recebido em: 18/10/99

Aceito em: 04/10/00 